\title{
Adoecimentos e afastamentos laborais em trabalhadores municipais: Um estudo transversal no extremo sul do Brasil
}

\author{
Sickness and absence from work among municipal workers: A cross-sectional study in the extreme \\ south of Brazil \\ Enfermedad y absentismo laboral entre trabajadores municipales: Un estudio transversal en el \\ extremo sur de Brasil
}

\section{Resumo}

Objetivo: Identificar os afastamentos laborais, concedidos por licenças saúde, justificados por adoecimentos e agravos de trabalhadores de secretarias do setor público municipal, no extremo sul do Brasil. Metodologia: Trata-se de uma pesquisa transversal descritiva e analítica, com abordagem quantitativa. A coleta de dados foi extraída de um banco do Setor de Saúde Ocupacional do município do estudo. Na análise prévia dos dados, foi aplicada a estatística descritiva. Por se tratar de dados secundários, foram respeitadas as diretrizes éticas do Conselho Nacional de Saúde. Resultados: Dos 4.120 afastamentos laborais dos servidores municipais, por licença saúde, $52,6 \%$ foram de servidores professores; constatou-se que, não obstante os afastamentos laborais, tenham sido justificados por até 6 diagnósticos, codificados segundo a classificação internacional de doenças, 95,6\% foram anotados por um diagnóstico; do conjunto de doenças anotadas nas licenças saúde, $68 \%$ se enquadraram em 5 grupos da classificação internacional das doenças: "Fatores que influenciam o estado de saúde e o contato com os serviços de saúde"; "Doenças do Sistema Osteomuscular e do Tecido Conjuntivo"; "Doenças do Aparelho Respiratório"; "Doenças do Aparelho Digestivo"; e "Sintomas, Sinais e Achados Anormais de Exames Clínicos e de Laboratório; quando considerado o código da categoria da classificação internacional, o enquadramento do conjunto de doenças abrangeu 367 categorias. Conclusão: Espera-se contribuir para o desenvolvimento de estratégias de gestão que visem a segurança e as condições saudáveis do trabalhador e dos ambientes laborais.

Palavras-chave: Absenteísmo; Setor público; Doenças profissionais; Saúde do trabalhador.

\section{Abstract}

Objective: To identify work leaves, granted by sick leave, justified by illnesses and injuries of workers from municipal public sector departments, in the extreme south of Brazil. Methodology: This is a cross-sectional descriptive and analytical research, with a quantitative approach. The data collection was extracted from a bank in the Occupational Health Sector in the municipality of the study. In the previous analysis of the data, descriptive statistics was applied. As 
these are secondary data, the ethical guidelines of the National Health Council were respected. Results: Of the 4,120 labor absenteeism of municipal employees, for sick leave, $52.6 \%$ were teachers servers; it was found that, despite absence from work, they were justified by up to 6 diagnoses, coded according to the international classification of diseases, $95.6 \%$ were recorded by a diagnosis; of the set of diseases noted in health licenses, $68 \%$ fell into 5 groups of the international classification of diseases: "Factors influencing health status and contact with health services"; "Diseases of the Musculoskeletal System and Connective Tissue"; "Respiratory System Diseases "; "Diseases of the Digestive System"; Symptoms, signs and abnormal clinical and laboratory findings, not elsewhere classified, when the category code from the international classification is taken into account, the framework of the set of diseases covered 367 categories. Conclusion: It is hoped to contribute to the development of management strategies for workers' and work environments' safety and healthy conditions.

Keywords: Absenteeism; Public sector; Occupational diseases; Occupational health.

\section{Resumen}

Objetivo: Identificar las bajas laborales, otorgadas por baja por enfermedad, justificadas por enfermedades y lesiones de los trabajadores de los departamentos del sector público municipal, en el extremo sur de Brasil. Metodología: Se trata de una investigación descriptiva y analítica transversal, con enfoque cuantitativo. La recolección de datos se extrajo de un banco del Sector Salud Ocupacional del municipio de estudio. En el análisis previo de los datos se aplicó estadística descriptiva. Al tratarse de datos secundarios, se respetaron las directrices éticas del Consejo Nacional de Salud. Resultados: De los 4.120 permiso de ausencia de los empleados municipales, por baja sanitaria, el 52,6\% eran profesorado; se encontró que, a pesar de la ausencia laboral, se justificaron hasta por 6 diagnósticos, codificados según la clasificación internacional de enfermedades, el 95,6\% fueron registrados por un diagnóstico; del conjunto de enfermedades señaladas en las licencias sanitarias, el 68\% se clasificó en 5 grupos de la clasificación internacional de enfermedades: "Factores que influyen en el estado de salud y el contacto con los servicios de salud"; "Enfermedades osteomusculares y del tejido conjuntivo"; "Enfermedades del sistema respiratorio "; "Enfermedades del sistema digestivo"; "; "Síntomas, signos y hallazgos clínicos y de laboratorio anormales, no clasificados en otra parte", al considerar el código de categoría de la clasificación internacional, la distribución del conjunto de enfermedades abarcaba 367 categorías. Conclusión: Se espera contribuir al desarrollo de estrategias de gestión para la seguridad y salud de los trabajadores y entornos laborales.

Palabras clave: Absentismo; Sector público; Salud laboral; Enfermedades profesionales.

\section{Introdução}

Na rede pública de atenção às necessidades sociais, os afastamentos laborais dos servidores por adoecimento e os respectivos processos de estabelecimento gradual das doenças e agravos, de forma geral, são subjugados aos determinantes sociais e a legislação que induz a forma de agir das pessoas, podendo ser acentuados pela volatilidade das relações laborais e ambientais. $\mathrm{O}$ acompanhamento e análise das características destes eventos favorece o monitoramento e a possibilidade de resolução tanto de casos iminentes, como dos casos estabelecidos, de doenças e agravos à saúde, sendo essenciais para a saúde pública. As doenças com o significado de enfermidades ou estados clínicos, independentemente de origem ou fonte, que representem ou possam representar danos significativos para os seres humanos e os agravos com significado de quaisquer danos à integridade física, mental e social dos indivíduos provocado por circunstâncias nocivas, como acidentes, intoxicações, abuso de drogas, e lesões auto ou heteroinfligidas (Brasil, 2014).

Recorrentes afastamentos laborais podem sinalizar para as condições de saúde dos trabalhadores, dos ambientes de trabalho e das atividades realizadas, potencializando aos gestores públicos alertas à saúde e segurança em nível individual e coletivo dos servidores e dos ambientes de trabalho, indispensáveis a continuidade e qualidade dos serviços prestados aos usuários dos serviços públicos, bem como, a necessidade de acompanhamento e avaliação da significativa influência administrativa, financeira e social quando esse conjunto se encontra, por exemplo, na gerência e gestão de pessoas em determinado contexto socioambiental de trabalho.

Como efeito, geram-se impactos negativos, que na perspectiva dos servidores públicos se exteriorizam, entre outras, na forma de afastamentos laborais motivados por problemas de saúde, abrangendo as doenças e os agravos que podem levar as doenças crônicas e as aposentadorias precoces, com o ônus ao erário público dos elevados índices de repasses pecuniários sobre os afastamentos laborais temporários e os afastamentos por invalidez, caracterizados por incapacidades dos segurados às 
atividades de trabalho (Santos et al., 2015).

Como informação, dados extraídos da Pesquisa Nacional de Saúde, em 2013, mostram que 45,1\% dos entrevistados informaram ter pelo menos uma doença crônica, sendo a Região Sul do país a de maior prevalência, com 52,1\% dos casos (Brasil, 2013). Dados da organização Brazil-US Business Council estimam que até o ano de 2030 os casos de absenteísmo e aposentadoria precoce no Brasil, relacionados a doenças crônicas adquiridas pelos trabalhadores, venham a impactar na redução de $8,7 \%$ do Produto Interno Bruto do país (Brasil, 2011).

O Sistema Único de Saúde (SUS) e seus componentes organizativos compõem uma rede hierárquica de assistência e organização em prol de garantir às pessoas e à coletividade condições de bem-estar físico, mental e social, na responsabilidade pública e corresponsabilidade privada - representada pela pessoa, família e empresas na sociedade - em garantir estados saudáveis (Brasil, 2013). A rede de atenção básica, como espaço avançado do SUS de organização do cuidado, onde a população de um determinado município tem acesso ao atendimento de suas demandas, inclui também a atenção à população trabalhadora. É um sítio relevante para a produção de indicadores da saúde do trabalhador. Além dos fatores epidemiológicos que envolvem o conhecimento da população e suas características, há que se destacar os avanços tecnológicos existentes e, com relevância das informações, sabe-se que poucos serviços utilizam os indicadores na tomada de decisões para melhorias do planejamento local em saúde visando a qualidade da assistência.

Neste contingente, evidencia-se a relevância de pesquisas que contemplem as necessidades fundamentais da população trabalhadora, na possibilidade de produzir evidências acerca da relação entre os processos de trabalho e as possíveis causas/efeitos nas condições de estados de saúde e doença de trabalhadores que acarretam prejuízo humano ao trabalhador, ao empregador e à sociedade. A utilização sistemática de indicadores como ferramenta na gestão da atenção à saúde tem contribuído para a redução de custos e de subsídio financeiro, visando o aumento da produtividade e da assistência resolutiva e de qualidade aos trabalhadores usuários do sistema, uma vez que agrega questões como território, situação problema e consciência ambiental.

Neste estudo buscou-se potencializar o uso de informações já disponíveis na estrutura organizacional e administrativa de um município localizado no extremo Sul do Brasil, onde o Núcleo de Assistência à Saúde Ocupacional (NASO) da prefeitura se constitui no segmento responsável pelo acolhimento das demandas do processo estabelecido de adoecimentos dos servidores municipais, incluindo os trabalhadores da própria Secretaria de Saúde. Ao proceder a inserção do cadastro para afastamento laboral de cada servidor, justificado pelos variados determinantes de saúde, gera-se um registro associado ao código da Classificação Internacional de Doenças (CID-10) (OMS, 2006), que é a variável geradora do afastamento do sítio de trabalho do servidor.

Assim, têm-se como objetivos: identificar os afastamentos laborais, concedidos por licenças saúde, justificadas por adoecimentos e agravos de trabalhadores de secretarias do setor público municipal, no Sul do Brasil; e caracterizar os afastamentos laborais identificados, contemplando informações como a data do apontamento administrativo da licença saúde concedida, a idade do trabalhador, o gênero, o setor de atuação (secretaria de município), a profissão vinculada a atuação (cargo de trabalho), os dias de licença, as doenças relatadas nas licenças médicas e codificação das doenças, de acordo com a classificação internacional das doenças (CID-10).

\section{Metodologia}

\subsection{Delineamento da pesquisa}

Esta pesquisa caracteriza-se como um estudo descritivo, analítico e exploratório, com abordagem quantitativa de dados secundários (Vieira, 2011), aplicado ao tema "afastamentos laborais, em decorrência de adoecimentos e agravos, em servidores de um município, localizado no extremo sul do Brasil". Faz parte, em seus objetivos e compromissos, ao macroprojeto intitulado 
"Dimensão socioambiental e atenção básica no sul do Brasil: saúde do servidor/trabalhador, riscos, acidentes e doenças relacionadas ao trabalho".

\subsection{Aspectos éticos}

Por se tratar de uma pesquisa com utilização de dados secundários, referentes a episódios de afastamentos laborais de trabalhadores públicos municipais, foram assegurados o anonimato e o sigilo dos dados analisados, conforme Resolução do Conselho Nacional de Saúde, n 466/12 (Brasil, 2012). Em seus objetivos e compromissos o presente estudo foi aprovado no Comitê de Ética da Universidade Federal do Rio Grande (CEP - FURG), sob parecer n $\mathrm{n}^{\circ}$ 122/2017e CAAE: 70043717.0.0000.5324.

\subsection{Dados da pesquisa}

Os dados da pesquisa foram obtidos de fonte secundária, a partir de planilhas com informações de controle administrativo, constantes dos arquivos digitais, de guarda de documentos, do Núcleo de Assistência à Saúde Ocupacional (NASO), da Secretaria de Gestão Administrativa do município, definido como local do estudo. Tendo por referência o ano de 2018 foram disponibilizadas ao estudo 12 planilhas, uma para cada mês do ano, caracterizando, no total, 4.427 registros de concessões de licenças para afastamento do trabalho, concedidas, aos servidores municipais para tratamento de saúde, pelo serviço médico do município. Cada registro contendo as informações sobre: idade do servidor; gênero; setor de atuação (secretaria de município); profissão de vínculo de atuação (cargo de trabalho); datas de início e fim do afastamento do trabalho, data do apontamento administrativo da licença saúde concedida; dias de licença; doenças relatadas na licença médica, com a codificação do adoecimento e/ou agravo, de acordo com a CID-10. O efetivo de servidores no município, no período pesquisado, foi estimado em 4.556 trabalhadores ativos.

\subsection{Organização dos dados}

Os dados incluídos nas planilhas facultadas ao estudo, foram organizados em uma tabela Excel, com linhas correspondendo as licenças e colunas correspondendo as informações. Cada registro (linha da tabela com as informações da licença concedida) foi examinado quanto as condições de completude e coerência das informações, como critérios para inclusão no banco de dados da pesquisa. A opção por não acrescer informações aos registros, além das já constantes nas planilhas, e a decisão de descartar os registros incompletos, fez com que 307 registros fossem excluídos, resultando um banco de dados na pesquisa com 4.120 linhas, melhor dizendo, registros de afastamentos do trabalho, por licenças de saúde. Observou-se, também, na organização das informações sobre as doenças relatadas nas licenças médicas, a existência de registros em que as causas dos afastamentos foram anotadas por extenso, com o nome comum do adoecimento e agravos, ao invés do código CID-10. A opção foi por manter os registros, substituindo as anotações por extenso pelos códigos da categoria da CID-10, em que se enquadravam. O banco de dados do estudo foi, então, estruturado a partir das informações constantes na tabela Excel referida acima, no destaque das variáveis disponíveis para o estudo.

\subsection{Análise dos dados}

Os dados da pesquisa foram analisados com o auxílio do software SPSS (Statistical Package for the Social Sciences) versão 22. No ambiente do editor de dados do SPSS foram incluídas as variáveis do estudo e digitados os dados correspondentes, sendo usada a estatística descritiva para descrever, resumir e apresentar os dados, por tabelas e gráficos, de forma a viabilizar e facilitar o processo de análises do estudo. Nas análises, a variável "idade dos servidores" foi definida por 6 faixas etárias, a variável "setor de atuação" foi definida por 20 secretarias do município do estudo e a variável "profissão de vínculo de atuação" 
foi definida por 48 cargos, do quadro de servidores do município. A variável "doenças relatas na licença saúde” foi definida pelo código da CID-10, considerando duas situações: o enquadramento pelo código do grupo da CID-10; e o enquadramento pelo código da categoria da CID-10. Não foi considerado no estudo o enquadramento das doenças pelo código da subcategoria da CID-10. Também, no caso de licenças saúde apontadas com mais de uma doença, para compor a relação de doenças no estudo, foi considerado o enquadramento na CID-10 da primeira doença apontada.

\section{Resultados}

Os avanços do estudo corresponderam as análises feitas às variáveis do banco de dados da pesquisa, composto por 4.120 registros de licenças médicas, para afastamento do trabalho de 1.525 servidores municipais no sul do Brasil, os quais estiveram envolvidos com estas licenças, no ano de 2018. Assim, a seguir no texto apresentam-se os resultados do estudo, em termos e no contexto da descrição das distribuições de frequência dos arranjos de valores, do banco de dados, que definem as variáveis do estudo.

Os 4.120 registros do banco de dados do estudo foram organizados quanto ao mês do apontamento administrativo da licença saúde concedida, gerando a distribuição de frequência apresentada na Tabela 1. O mês de agosto mostrou-se como de maior frequência. Os meses de dezembro, janeiro e fevereiro foram os de menor frequência, não superando juntos a frequência de agosto.

Tabela 1 - Frequência dos apontamentos administrativos de afastamentos laborais por mês do ano de 2018, correspondentes a licenças saúde de servidores municipais. Região Sul, Brasil.

\begin{tabular}{l|c|c|c}
\hline \multicolumn{1}{c|}{ Mês } & Frequência & $\begin{array}{c}\text { Porcentagem } \\
(\boldsymbol{\%})\end{array}$ & $\begin{array}{c}\text { Porcentagem } \\
\text { Acumulativa (\%) }\end{array}$ \\
\hline Janeiro & 97 & 2,4 & 2,4 \\
\hline Fevereiro & 130 & 3,2 & 5,5 \\
\hline Março & 316 & 7,7 & 13,2 \\
\hline Abril & 353 & 8,6 & 21,7 \\
\hline Maio & 465 & 11,3 & 33,0 \\
\hline Junho & 395 & 9,6 & 42,6 \\
\hline Julho & 388 & 9,4 & 52,0 \\
\hline Agosto & 604 & 14,7 & 66,7 \\
\hline Setembro & 390 & 9,5 & 76,2 \\
\hline Outubro & 381 & 9,2 & 85,4 \\
\hline Novembro & 393 & 9,5 & 95,0 \\
\hline Dezembro & 208 & 5,0 & 100,0 \\
\hline \multicolumn{1}{c|}{ Total } & $\mathbf{4 1 2 0}$ & $\mathbf{1 0 0 , 0}$ & \\
\hline
\end{tabular}

Fonte: Banco de dados da pesquisa (2021)

Os dados referentes ao sexo dos servidores afastados do trabalho por licença saúde foram organizados pela distribuição de frequência dada na Tabela 2. O sexo feminino foi prevalente nas licenças saúde concedidas aos servidores municipais. 
Tabela 2 - Frequência das informações sobre gênero nos afastamentos laborais por sexo do servidor em licença saúde no município. Região Sul, Brasil, 2018.

\begin{tabular}{|c|c|c|c|}
\hline Sexo & Frequência & $\begin{array}{c}\text { Porcentagem } \\
(\%)\end{array}$ & $\begin{array}{c}\text { Porcentagem } \\
\text { Acumulativa (\%) }\end{array}$ \\
\hline Feminino & 3406 & 82,7 & 82,7 \\
\hline Masculino & 714 & 17,3 & 100,0 \\
\hline Total & 4120 & 100,0 & \\
\hline
\end{tabular}

Fonte: Banco de dados da pesquisa (2021)

A Tabela 3 mostra a destruição de frequência que foi obtida da organização dos dados sobre a idade dos servidores em 5 faixas etárias. Observa-se que $64,4 \%(34,2 \%+30,2 \%)$ dos afastamentos do trabalho, pelos servidores em licença saúde no município estudado, correspondeu a indivíduos produtivos, com idade entre 31 e 50 anos. A maior frequência, 34,2\%, contemplou indivíduos produtivos com idade entre 31 e 40 anos.

Tabela 3 - Frequência da idade dos servidores municipais com afastamento laboral por faixa etária. Região Sul, Brasil, 2018.

\begin{tabular}{c|c|c|c}
\hline Fa & Frequência & $\begin{array}{c}\text { Porcentagem } \\
\mathbf{( \% )}\end{array}$ & $\begin{array}{c}\text { Porcentagem } \\
\text { Acumulativa (\%) }\end{array}$ \\
\hline De 20 a 30 anos & 219 & 5,3 & 5,3 \\
\hline De 31 a 40 anos & 1409 & 34,2 & 39,5 \\
\hline De 41 a 50 anos & 1243 & 30,2 & 69,7 \\
\hline De 51 a 60 anos & 1026 & 24,9 & 94,6 \\
\hline De 61 a 70 anos & 209 & 5,1 & 99,7 \\
\hline De 71 a 75 anos & 14 &, 3 & 100,0 \\
\hline Total & $\mathbf{4 1 2 0}$ & $\mathbf{1 0 0 , 0}$ & \\
\hline
\end{tabular}

Fonte: Banco de dados da pesquisa (2021).

A Figura 1 apresenta a distribuição de frequência que foi obtida das informações sobre o enquadramento, no quadro de secretarias do município, dos servidores licenciados do trabalho organizadas em ordem crescente do número de registros. Observa-se que foram 25 secretarias municipais que tiveram servidores afastados do trabalho, sendo que a Secretaria de Município da Educação (SMED) foi a que mais contribui para os afastamentos do trabalho, com 55,2\% dos casos. A segunda maior contribuição foi da Secretaria de Município da Saúde (SMS), respondendo por 20,3\% dos casos. Juntas, as 2 secretarias foram responsáveis por 75,5\% $(55,2 \%+20,3 \%)$ dos afastamentos do trabalho, por licença saúde dos servidores municipais, no estudo. 
Figura 1 - Frequência do enquadramento dos servidores licenciados do trabalho por secretarias do município, organizada em ordem crescente do número de registros no banco de dados do estudo. Região Sul, Brasil, 2018.

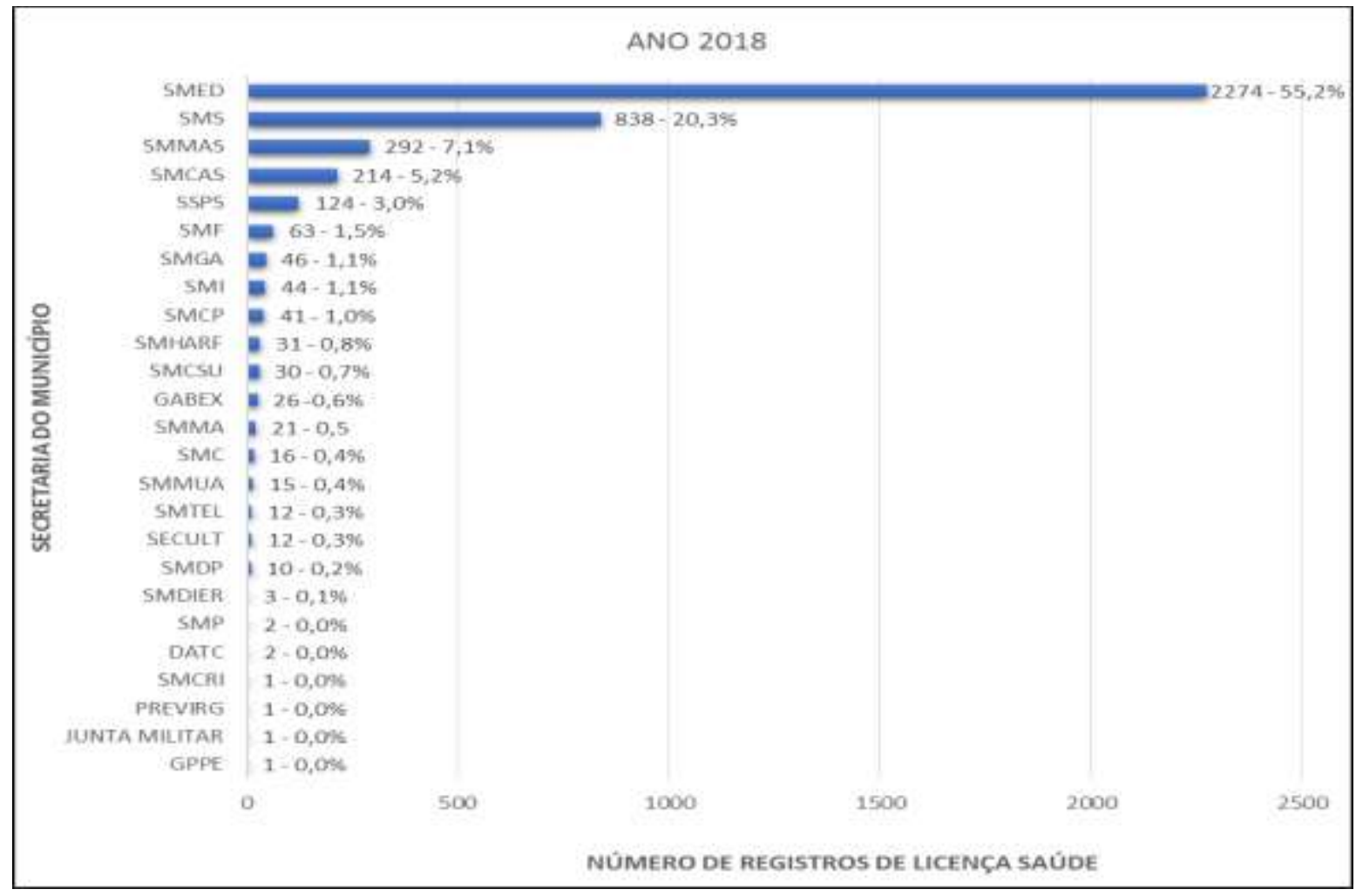

Fonte: Banco de dados da pesquisa (2021).

As informações sobre a profissão dos servidores municipais, que corresponde ao cargo de trabalho anotado na concessão da licença saúde, foram agrupadas na Tabela 4. Observa-se que os professores representaram 52,6\% dos casos, o que se mostra compatível com o envolvimento nas licenças saúde da Secretaria de Município da Educação (SMED), onde se vincularam os docentes, como acima destacado no texto e apresentado na Figura 1.

Tabela 4 - Frequência dos cargos de trabalho dos servidores municipais em afastamento laboral decorrentes da concessão de licenças saúde. Região Sul, Brasil, 2018.

\begin{tabular}{l|c|c|c}
\hline \multicolumn{1}{c|}{ Cargo } & Frequência & $\begin{array}{c}\text { Porcentagem } \\
(\%)\end{array}$ & $\begin{array}{c}\text { Porcentagem } \\
\text { Acumulativa (\%) }\end{array}$ \\
\hline Agente Comunitário em Saúde & 158 & 3,8 & 3,8 \\
\hline Agente de Trânsito e Transportes & 246 & 6,0 & 9,8 \\
\hline Agente Vigilante em Saúde & 6 &, 1 & 10,0 \\
\hline Arquiteto & 37 &, 9 & 10,8 \\
\hline Assessor Administrativo & 83 & 2,0 & 12,9 \\
\hline Assistente Social & 61 & 1,5 & 14,3 \\
\hline Atendente em Saúde & 51 & 1,2 & 15,6 \\
\hline Atendente Social & 2 &, 0 & 15,6 \\
\hline Auditor Fiscal & 13 &, 3 & 15,9 \\
\hline Auxiliar Administrativo & 16 &, 4 & 16,3 \\
\hline Bibliotecário & 25 &, 6 & 16,9 \\
\hline Carpinteiro & 1 &, 0 & 17,0 \\
\hline
\end{tabular}


Research, Society and Development, v. 10, n. 5, e50810515141, 2021

(CC BY 4.0) | ISSN 2525-3409 | DOI: http://dx.doi.org/10.33448/rsd-v10i5.15141

\begin{tabular}{|c|c|c|c|}
\hline Contador & 9 & ,2 & 17,2 \\
\hline Desenhista & 10 & ,2 & 17,4 \\
\hline Economista & 6 &, 1 & 17,6 \\
\hline Educador Físico & 25 & ,6 & 18,2 \\
\hline Educador Social & 121 & 2,9 & 21,1 \\
\hline Eletricista & 18 & ,4 & 21,6 \\
\hline Enfermeiro & 112 & 2,7 & 24,3 \\
\hline Engenheiro & 33 &, 8 & 25,1 \\
\hline Extra Classe & 21 &, 5 & 25,6 \\
\hline Farmacêutico & 1 &, 0 & 25,6 \\
\hline Fiscal Ambiental & 2 &, 0 & 25,7 \\
\hline Fiscal de Serviços & 31 &, 8 & 26,4 \\
\hline Fisioterapeuta & 11 &, 3 & 26,7 \\
\hline Fonoaudiólogo & 7 & ,2 & 26,8 \\
\hline Guarda Municipal & 134 & 3,3 & 30,1 \\
\hline Jornalista & 1 &, 0 & 30,1 \\
\hline Mecânico & 2 &, 0 & 30,2 \\
\hline Médico & 78 & 1,9 & 32,1 \\
\hline Monitor & 31 &, 8 & 32,8 \\
\hline Motorista & 61 & 1,5 & 34,3 \\
\hline Nutricionista & 38 & ,9 & 35,2 \\
\hline Odontólogo & 42 & 1,0 & 36,2 \\
\hline Operador de Máquinas & 14 &, 3 & 36,6 \\
\hline Operário & 157 & 3,8 & 40,4 \\
\hline Professor & 2168 & 52,6 & 93,0 \\
\hline Psicólogo & 38 & ,9 & 93,9 \\
\hline Secretário de Escola & 31 & ,8 & 94,7 \\
\hline Técnico Administrativo & 27 &, 7 & 95,3 \\
\hline Técnico Agrícola & 3 &, 1 & 95,4 \\
\hline Técnico em Enfermagem & 160 & 3,9 & 99,3 \\
\hline Técnico em Saúde & 10 &, 2 & 99,5 \\
\hline Técnico em Segurança do Trabalho & 2 &, 0 & 99,6 \\
\hline Técnico Superior em Máquinas & 7 & ,2 & 99,8 \\
\hline Turismólogo & 4 & ,1 & 99,9 \\
\hline Veterinário & 5 &, 1 & 100,0 \\
\hline Vigilante Sanitário & 1 &, 0 & 100,0 \\
\hline Total & 4120 & 100,0 & \\
\hline
\end{tabular}

Fonte: Banco de dados da pesquisa (2021)

Os dados referentes ao número de dias de afastamento do trabalho, nas licenças saúde concedidas aos servidores municipais, constantes no banco de dados da pesquisa, foram organizados quanto a duração de cada licença, em dias, gerando uma distribuição de frequência mostrada, de forma resumida, na Tabela 5. Observando os números desta tabela, os afastamentos 
laborais de 1 dia representaram 57,1\% dos afastamentos laborais no município, enquanto os afastamentos laborais de mais de um dia contabilizaram $42,9 \%$ deste total.

Tabela 5 - Frequência da duração dos afastamentos laborais dos servidores municipais por 1dia ou mais de 1 dia de licença saúde, concedidas pelo setor médico do município. Região Sul, Brasil, 2018.

\begin{tabular}{|c|c|c|c|}
\hline Duração da Licença & Frequência & $\begin{array}{c}\text { Porcentagem } \\
(\%)\end{array}$ & $\begin{array}{c}\text { Porcentagem } \\
\text { Acumulativa (\%) }\end{array}$ \\
\hline Mais de 1 dia & 1767 & 42,9 & 42,9 \\
\hline 1 dia & 2353 & 57,1 & 100 \\
\hline Total & 4120 & 100,0 & 100 \\
\hline
\end{tabular}

Fonte: Banco de dados da pesquisa (2021).

Contribuindo com as informações sobre os dias de afastamento do trabalho dos servidores municipais nas licenças saúde, considerou-se, na tabela 6 , a contabilização do total de dias de afastamentos laborais, somando as durações das licenças. Observa-se que no período do estudo, as licenças saúde foram responsáveis por 24.387 ausências ao trabalho dos servidores municipais. As licenças de 1 dia que, como antes referido no texto, foram responsáveis por mais da metade dos registros de afastamentos laborais, representaram apenas 9,6\% do total geral de dias contabilizados para todos os afastamentos laborais dos servidores municipais, no período do estudo.

Tabela 6 - Número total de dias de afastamento laboral dos servidores municipais pela duração das licenças saúde, concedidas pelo setor médico do município. Região Sul, Brasil, 2018.

\begin{tabular}{|c|c|c|c|c|c|}
\hline $\begin{array}{c}\text { Duração } \\
\text { da Licença } \\
\text { (Dias) }\end{array}$ & Frequência & $\begin{array}{l}\text { Total de } \\
\text { Dias }\end{array}$ & $\begin{array}{c}\text { Dias } \\
\text { Acumulados }\end{array}$ & \begin{tabular}{|c|}
$\begin{array}{c}\text { Porcentagem } \\
\text { do Total de Dias* } \\
(\%)\end{array}$ \\
\end{tabular} & $\begin{array}{c}\text { Porcentagem } \\
\text { Acumulativa } \\
(\%)\end{array}$ \\
\hline 1 & 2353 & 2353 & 2353 & 9,6 & 9,6 \\
\hline 2 & 488 & 976 & 3329 & 4,0 & 13,7 \\
\hline 3 & 187 & 561 & 3890 & 2,3 & 16,0 \\
\hline 4 & 231 & 924 & 4814 & 3,8 & 19,7 \\
\hline 5 & 56 & 280 & 5094 & 1,1 & 20,9 \\
\hline 6 & 141 & 846 & 5940 & 3,5 & 24,4 \\
\hline 7 & 49 & 343 & 6283 & 1,4 & 25,8 \\
\hline 8 & 13 & 104 & 6387 & 0,4 & 26,2 \\
\hline 9 & 127 & 1143 & 7530 & 4,7 & 30,9 \\
\hline 10 & 16 & 160 & 7690 & 0,7 & 31,5 \\
\hline 11 & 33 & 363 & 8053 & 1,5 & 33,0 \\
\hline 12 & 5 & 60 & 8113 & 0,2 & 33,3 \\
\hline 13 & 115 & 1495 & 9608 & 6,1 & 39,4 \\
\hline 14 & 236 & 3304 & 12912 & 13,5 & 52,9 \\
\hline 15 & 4 & 60 & 12972 & 0,2 & 53,2 \\
\hline 31 & 1 & 31 & 13003 & 0,1 & 53,3 \\
\hline 32 & 1 & 32 & 13035 & 0,1 & 53,5 \\
\hline 33 & 1 & 33 & 13068 & 0,1 & 53,6 \\
\hline 119 & 2 & 238 & 13306 & 1,0 & 54,6 \\
\hline 148 & 1 & 148 & 13454 & 0,6 & 55,2 \\
\hline 149 & 1 & 149 & 13603 & 0,6 & 55,8 \\
\hline 179 & 50 & 8950 & 22553 & 36,7 & 92,5 \\
\hline
\end{tabular}


Research, Society and Development, v. 10, n. 5, e50810515141, 2021

(CC BY 4.0) | ISSN 2525-3409 | DOI: http://dx.doi.org/10.33448/rsd-v10i5.15141

\begin{tabular}{c|c|c|c|c|c}
\hline 180 & 7 & 1260 & 23813 & 5,2 & 97,6 \\
\hline 207 & 1 & 207 & 24020 & 0,8 & 98,5 \\
\hline 367 & 1 & 367 & 24387 & 1,5 & 100,0 \\
\hline TOTAL & $\mathbf{4 1 2 0}$ & $\mathbf{2 4 3 8 *}$ & & $\mathbf{1 0 0}$ & \\
\hline
\end{tabular}

*Total geral de dias de afastamento do trabalho no ano de 2018. Fonte: Banco de dados da pesquisa (2021)

Continuando a observar os números da Tabela 6, as ausências ao trabalho de até 12 dias representaram um terço (33,3\%) do total geral anual de ausências ao trabalho, enquanto os afastamentos do trabalho de até 14 dias representaram mais da metade $(52,9 \%)$ deste total geral anual de ausências ao trabalho. Foram poucos os afastamentos com duração acima de 15 dias, mas representaram a outra quase metade $(47,1 \%)$ do total geral anual de ausências ao trabalho. Os afastamentos de até 14 dias se mostraram frequentes, da mesma forma que os afastamentos com maior duração se mostraram eventuais, mas ambos impactaram o número total anual de dias de afastamento quase na mesma proporção.

As doenças referidas nas licenças médicas foram organizadas quanto ao número de doenças informadas em cada registro de licença. Quantitativo este que foi obtido para cada registro, a partir da codificação de cada adoecimento e agravos de acordo com a categoria da CID-10. A Tabela 7 mostra que, embora tenham ocorridos registros de licenças justificadas com até 6 doenças, a predominância, com 3.939 (95,6\%) do total de casos, foi dos registros justificados com 1 doença.

Tabela 7 - Frequência do número de doenças referidas em cada licença saúde para justificar o afastamento laboral do servidor municipal. Região Sul, Brasil, 2018.

\begin{tabular}{c|r|r|r}
\hline $\begin{array}{c}\text { Número } \\
\text { de } \\
\text { Doenças }\end{array}$ & \multicolumn{1}{|c|}{ Frequência } & $\begin{array}{c}\text { Porcentagem } \\
(\%)\end{array}$ & \multicolumn{2}{c}{$\begin{array}{c}\text { Porcentagem } \\
\text { Acumulativa (\%) }\end{array}$} \\
\hline 1 & 3939 & 95,6 & 95,6 \\
\hline 2 & 156 & 3,8 & 99,4 \\
\hline 3 & 17 &, 4 & 99,8 \\
\hline 4 & 6 &, 1 & 100,0 \\
\hline 6 & 2 &, 0 & 100,0 \\
\hline Total & $\mathbf{4 1 2 0}$ & $\mathbf{1 0 0 , 0}$ & \\
\hline
\end{tabular}

Fonte: Banco de dados da pesquisa (2021).

Os 4.120 registros no banco de dados do estudo, referente aos adoecimentos e agravos que justificaram as licenças médicas, para concessões de afastamentos do trabalho aos servidores municipais do estudo, foram organizadas quanto ao enquadramento das doenças pelos códigos relativos aos grupos da CID-10.

A Figura 2 mostra o grupo (Z00-Z99), que agrega as causas dos adoecimentos e agravos decorrentes de "Fatores que Influenciam o Estado de Saúde e o Contato com os Serviços de Saúde", como predominante nos registros das licenças saúde, com 19\% dos casos. Junto com os outros 4 grupos, na ordem decrescente do predomínio, somam 68\% dos casos: o grupo (M00M99), referente as "Doenças do Sistema Osteomuscular e do Tecido Conjuntivo"; o grupo (J00-J99), referente as "Doenças do Aparelho Respiratório"; o grupo (K00-K93), referente as "Doenças do Aparelho Digestivo"; e o grupo (R00-R99), referente aos "Sintomas, Sinais e Achados Anormais de Exames Clínicos e de Laboratório, Não Classificados em Outra Parte”. 
Figura 2 - Frequência das doenças informadas nas licenças saúde concedidas aos servidores municipais por grupo da CID-10. Região Sul, Brasil, 2018.

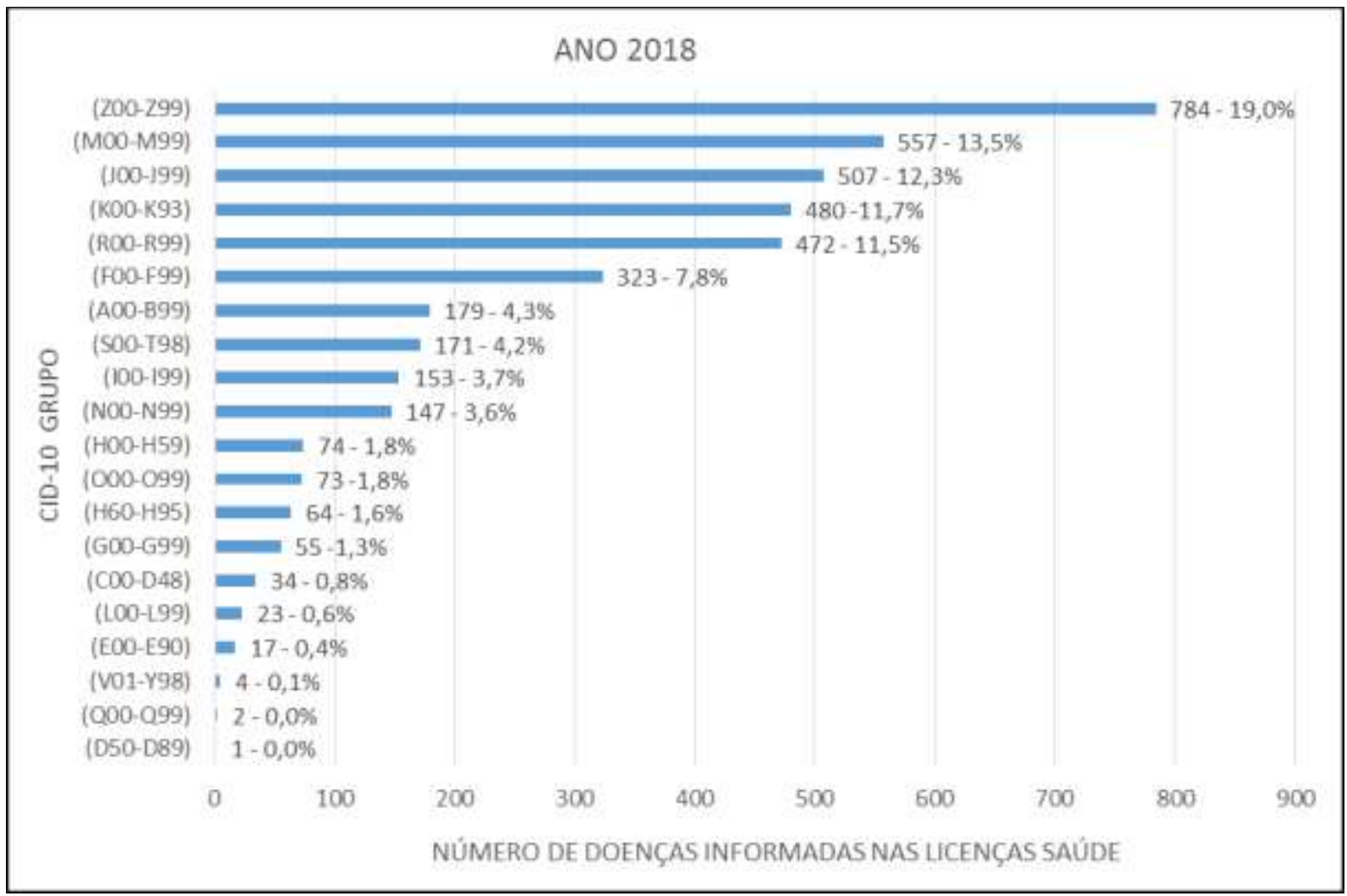

Fonte: Banco de dados da pesquisa (2021).

Para complementar as informações referente aos adoecimentos e agravos, os 4.120 registros no banco de dados do estudo, que justificaram as licenças médicas, para concessões de afastamentos do trabalho aos servidores municipais do estudo, foram organizados, agora, quanto ao enquadramento das doenças pelos códigos relativos as categorias da CID-10. A distribuição de frequências obtida, disposta em ordem decrescente dos valores, permitiu ranquear as categorias da CID-10, no estudo. Também, mostrou que as frequências se distribuíram por 367 diferentes categorias da CID-10. Na tabela 8 se destacam 20 destas categorias, as de maior frequência.

A contabilização das frequências, correspondentes as categorias da CID-10, mostrou que os 20 destaques da tabela 8 justificaram $64,2 \%$ do total de casos de afastamentos laborais, por licença saúde, no estudo. A predominância foi da categoria Z00, das causas de afastamento enquadradas como "Exame Geral e Investigação de Pessoas Sem Queixas ou Dia gnóstico Relatado". 
Tabela 8 - Frequência das doenças, nominadas segundo a categoria CID-10, referidas nas licenças saúde para justificar os afastamentos laborais dos servidores municipais. Região Sul, Brasil, 2018.

\begin{tabular}{|c|c|c|c|c|}
\hline $\begin{array}{l}\text { CID-10 } \\
\text { Categoria }\end{array}$ & $\begin{array}{l}\text { Nome da doença } \\
\text { (Categoria da CID-10) }\end{array}$ & Frequência* & $\begin{array}{l}\text { Porcentagem } \\
(\%)\end{array}$ & $\begin{array}{c}\text { Porcentagem } \\
\text { Acumulativa } \\
(\%)\end{array}$ \\
\hline Z00 & $\begin{array}{l}\text { Exame Geral e Investigação de Pessoas Sem } \\
\text { Queixas ou Diagnóstico Relatado }\end{array}$ & 604 & 14,7 & 14,7 \\
\hline R69 & $\begin{array}{l}\text { Causas Desconhecidas e Não Especificadas de } \\
\text { Morbidade }\end{array}$ & 378 & 9,2 & 23,9 \\
\hline M54 & Dorsalgia & 279 & 6,8 & 30,7 \\
\hline K08 & $\begin{array}{l}\text { Outros Transtornos Dos Dentes e de Suas } \\
\text { Estruturas de Sustentação }\end{array}$ & 215 & 5,2 & 35,9 \\
\hline $\mathrm{J} 11$ & $\begin{array}{l}\text { Influenza (gripe) Devida a Vírus Não } \\
\text { Identificado }\end{array}$ & 193 & 4,6 & 40,5 \\
\hline A09 & $\begin{array}{l}\text { Diarréia e Gastroenterite de Origem Infecciosa } \\
\text { Presumível }\end{array}$ & 145 & 3,5 & 44,0 \\
\hline F32 & Episódios Depressivos & 132 & 3,2 & 47,2 \\
\hline F41 & Outros Transtornos Ansiosos & 89 & 2,2 & 49,4 \\
\hline $\mathrm{I} 10$ & Hipertensão Essencial (primária) & 80 & 1,9 & 51,3 \\
\hline $\mathrm{J} 03$ & Amigdalite Aguda & 73 & 1,8 & 53,1 \\
\hline $\mathrm{Z} 34$ & Supervisão de Gravidez Normal & 69 & 1,7 & 54,8 \\
\hline $\mathrm{N} 30$ & Cistite & 61 & 1,5 & 56,3 \\
\hline M51 & Outros Transtornos de Discos Intervertebrais & 53 & 1,2 & 57,5 \\
\hline J06 & $\begin{array}{l}\text { Infecções Agudas Das Vias Aéreas Superiores } \\
\text { de Localizações Múltiplas e Não Especificadas }\end{array}$ & 42 & 1,0 & 58,5 \\
\hline $\mathrm{H} 83$ & Outros Transtornos do Ouvido Interno & 41 & 1,0 & 59,5 \\
\hline J01 & Sinusite Aguda & 40 & 1,0 & 60,5 \\
\hline M65 & Sinovite e Tenossinovite & 40 & 1,0 & 61,5 \\
\hline $\mathrm{H} 10$ & Conjuntivite & 39 & 0,9 & 62,4 \\
\hline $\mathrm{Z54}$ & Convalescença & 37 & 0,9 & 63,3 \\
\hline F43 & $\begin{array}{l}\text { "Reações ao ""stress"" Grave e Transtornos de } \\
\text { Adaptação" }\end{array}$ & 36 & 0,9 & 64,2 \\
\hline \multicolumn{2}{|l|}{ TOTAL } & $2646 * *$ & 64,2 & \\
\hline
\end{tabular}

*Frequência relativa ao número total de registros de licença saúde no estudo (4120 casos)

**Quantitativo que representa 64,2\% dos 4120 registros de licenças saúde dos servidores municipais

Fonte: Banco de dados da pesquisa (2021).

Pela importância atribuída aos transtornos mentais, observada na bibliografia, como uma das principais causas de ausência ao trabalho devido à doenças e incapacidades, buscou-se identificar, na tabela 8, as categorias da CID-10 explicitamente associadas a estas alterações do funcionamento da mente, que prejudicam o desempenho dos indivíduos. Foram 3 categorias identificadas, todas do grupo (F00-F99) - dos "Transtornos mentais e comportamentais" da CID-10: a categoria dos "Episódios Depressivos", a categoria dos "Outros Transtornos Ansiosos" e a categoria das "Reações ao "'stress"'" Grave e Transtornos de Adaptação".

As 3 categorias contabilizam $257(132+89+36)$ apontamentos em licenças saúde, no total de 4.120 registros de afastamento do trabalho no estudo, melhor dizendo $6,2 \%$ do total de casos. Informação esta, que não se mostrou suficiente para caracterizar os transtornos metais como predominantes, entre os motivos de afastamentos laborais, no estudo.

\section{Discussão}

O presente estudou analisou um conjunto de dados de uma base preliminar de informações disponibilizada pelo setor de registros e informações de um município no Sul do Brasil, com todos os profissionais (servidores municipais) que, por motivo 
de doenças e agravos, necessitaram de afastamento laboral, pelo menos uma vez no período do estudo. Pode-se, de imediato, salientar que foi possível identificar profissionais com maior tendência em apresentar necessidade de afastamento laboral do que outros, bem como a distribuição dos eventos pelas diversas secretarias do município.

Os resultados mostraram que, do conjunto de 4120 registros de afastamentos laborais de trabalhadores do setor público do município estudado, a prevalência foi do sexo feminino e da faixa etária entre 31 a 60 anos. Um estudo realizado com trabalhadores municipais na Finlândia (Vuorio et al., 2019) encontrou resultados semelhantes ao do presente estudo, ou seja, a maior frequência de afastamentos ocorreu no sexo feminino, quando comparada aos afastamentos do sexo masculino. Nesse estudo finlandês, entre vários fatores avaliados em relação ao trabalho, a autopercepção da capacidade para o trabalho mostrouse como um dos fatores mais significativos que afetam a taxa de absenteísmo. Ainda, revelou a capacidade para o trabalho prejudicada pela associação a doenças crônicas, afastamentos por doença e aposentadorias precoces. Destaca-se que o desenho de estudo aplicado na referência, não foi efetivado neste estudo, não obstante, seus resultados vão contribuir para o aprofundamento do estudo, no grupo de trabalhadores do setor público local.

Outra pesquisa realizada no Brasil, pelo pelos pesquisadores Leão et al. (2015), também se aproxima aos achados do presente estudo, por mostrar que a maioria dos afastamentos laborais se associa ao sexo feminino e que essa maioria se identifica com o setor da educação. Decerto na pesquisa referida acima o contingente de profissionais da área da educação tenha elevando quantitativo nos afastamentos laborais, como verificou-se neste estudo. Por outro lado, pesquisa realizada com trabalhadores do setor público italiano evidenciou redução de afastamento para o sexo feminino (Casimirri et al., 2014).

No que se refere à faixa etária, de forma semelhante ao achado neste estudo, algumas publicações nacionais (Santos \& Mattos, 2010) e internacionais (Reis et al., 2011) relatam tendência do aumento de episódios de afastamentos a partir do aumento da idade. Em outras palavras, os episódios de afastamentos laborais encontram-se presentes em trabalhadores nas faixas de idade produtivas, com prejuízos individuais e coletivos para a qualidade de vida e de bem-estar no trabalho. O degaste orgânico natural e o período de exposição às condições de trabalho são causas que podem explicar a maior prevalência de afastamentos do trabalho de trabalhadores com maior idade, mas ainda ativos.

Segundo dados acerca do absenteísmo no estudo finlandês (Vuorio et al., 2019), antes referido, os trabalhadores com fator idade mais avançada, insatisfação com a situação financeira e grau de instrução reduzida são os mais propensos a ausências. Além disso, possuíam indicadores correlatos, como o tabagismo, estabelecimento de doenças crônicas e qualidade de vida aquém do ideal no contexto social, a mais de sintomatologias relacionadas a transtornos depressivos e distúrbios corporais associados aos hábitos alimentares.

A análise do conjunto de dados sobre os episódios de afastamento do trabalho no que se refere a duração em dias do afastamento, para até 1 dia ou mais que 1 dia, mostrou a prevalência dos afastamentos de 1 dia. Quando contabilizado o número total de dias de afastamentos dos servidores, no período do estudo, a contribuição dos afastamentos de um dia de duração foi pequena.

$\mathrm{Na}$ ênfase sobre as secretarias municipais com maior número de ocorrência de afastamento do trabalho, a Secretaria de Município de Educação (SMED) foi predominante, com mais da metade dos casos. Na particularidade dos episódios de afastamentos laborais de professores municipais, os estudos de Costa (2017), Santos \& Mattos (2010) e evidenciam a relação entre as condições de trabalho e as ausências ao trabalho por motivo de doença, principalmente de professores que atuam em sala de aula, mas, também, daqueles que atuam em cargos diretivos. Estudo desenvolvido em outro município brasileiro encontrou como fatores preditivos de afastamento por doença o sexo feminino, idade avançada, baixa escolaridade, ser profissional de saúde, trabalho múltiplo e tempo integral. (Leão, Barbosa-Branco, Turchi, Steenstra \&Cole, 2017)

As condições laborais enfrentadas pelos professores, no dia a dia do trabalho, podem levar a um desequilíbrio entre o trabalho e a saúde física e mental, resultando no desenvolvimento de estresse, transtornos ansiosos e episódios depressivos. Neste 
contexto, estudo anterior realizado por Cezar-Vaz et. al. (2015) identificou cinco principais condições de trabalho estressantes elencadas pelos professores do ensino fundamental, relacionadas na sua maioria aos salários defasados e o material de trabalho inadequado ou insuficiente. Completam a lista o tamanho da sala de aula inadequado para o número de alunos, excesso de atividades e levar trabalho para casa. Outras dificuldades do trabalho mencionadas pelos entrevistados foram à dificuldade de manter os alunos focados na sala de aula, falta de reconhecimento da profissão, ruído na escola, carga horária excessiva, demandas escolares e sociais e dificuldade de convívio com os colegas. Destaca-se, ainda, outro estudo brasileiro em que os distúrbios mentais foram mais comuns entre os profissionais da educação (Leão, Barbosa-Branco, Turchi, Steenstra \&Cole, 2017).

Um número expressivo de episódios de afastamentos laborais apresentou como justificativa para concessão da licença saúde 1 diagnóstico, nominado conforme a CID-10. Houve justificativas com até 6 diagnósticos. Tal evidência possui importância não somente para o tempo presente, mas para o acompanhamento longitudinal do grupo de trabalhadores municipais, visando produzir e confirmar ou não tendências de adoecimentos e, assim, viabilizar a estruturação de processos terapêuticos preventivos e promotores de saúde, buscando melhorar a condição dos trabalhadores e do trabalho e, consequente, promover a permanência e durabilidade no trabalho de maneira saudável.

Considerando o enquadramento das doenças nos grupos da CID-10 foi predominante nas justificativas de afastamentos laborais, o código de grupo (Z00-Z99), referente aos "Fatores que Influenciam o Estado de Saúde e o Contato com os Serviços de Saúde". Também, em ordem decrescente do predomínio, se destacaram o grupo (M00-M99), referente as "Doenças do Sistema Osteomuscular e do Tecido Conjuntivo", o grupo (J00-J99), referente as "Doenças do Aparelho Respiratório", o grupo (K00K93), referente as "Doenças do Aparelho Digestivo" e o grupo (R00-R99), referente aos "Sintomas, Sinais e Achados Anormais de Exames Clínicos e de Laboratório, Não Classificados em Outra Parte". Se considerado o enquadramento nas categorias da CID-10 foi predominante nas justificativas de afastamento o código da categoria Z00, referente a "Exame Geral e Investigação de Pessoas Sem Queixas ou Diagnóstico Relatado".

Aspecto relevante é o fato dos transtornos mentais serem identificados, na literatura, como uma das principais causas de ausência ao trabalho devido à doenças e incapacidades (Wang et al., 2014; Muschalla \& Linden, 2013; Virtanen, Siukola, Lipiäinen, Liukkonen, Pentti \& Vahtera, 2017), impactando a duração dos benefícios auxílio-doença (Barbosa-Branco, Bültmann \& Steenstra, 2012). Segundo um estudo realizado no Japão, entre os afastamentos mais frequentes, encontram-se aqueles relacionados à saúde mental. Esses também ocasionam um período de maior afastamento, como períodos superiores a 30 dias (Nishiura et al., 2017). Corrobora com essas informações uma pesquisa com base em dados Suecos, que identifica os fatores relacionados ao estigma como preditivos para a prorrogação do retorno ao posto de trabalho de trabalhadores da população em geral, afastados por transtornos mentais, incluindo fatores gerados por doenças mentais relacionadas ao próprio trabalho (Bryngelson et al., 2013).

Ainda que, neste estudo, na relação das 20 doenças mais frequentes, nominadas pelos respectivos códigos de enquadramento pela categoria da CID-10, 3 códigos explicitamente enquadraram doenças associadas a diagnósticos de transtornos mentais, como os "Episódios Depressivos", "Outros Transtornos Ansiosos" e "Reações ao "'"stress"' Grave e Transtornos de Adaptação", as informações e análises realizadas não foram suficientes para caracterizar este fenômeno biopsicossocial como predominante entre os motivos de afastamentos laborais dos servidores municipais. Deve-se atentar para um aprofundamento na especificidade desse fato, frente à própria dificuldade em alguns casos de identificar e mesmo acompanhar a confirmação ou negação de problemas dessa natureza. No entanto, são necessárias análises mais aprofundadas para entender o que está acontecendo em termos da relação entre adoecimento e trabalho, a fim de que intervenções eficientes possam ser propostas.

Os fatores individuais e ambientais relacionados aos diferentes eventos de licença saúde não foram caracterizados neste 
estudo, não obstante, vários estudos em diferentes ambientes de trabalho evidenciam que os fatores do ambiente de trabalho, incluindo trabalho por turnos, estresse no trabalho e demandas de trabalho estão altamente correlacionados com a saúde e o bemestar dos trabalhadores (Theorell et al, 2015; Furlan, 2012; Costa, 2010). As doenças relacionadas ao trabalho causam dias de trabalho perdidos, aumentam o absenteísmo e reduzem a produtividade.

No processo da pesquisa realizada este estudo justificou-se pela finalidade de divulgar seus resultados, sem prejuízos à individualidade ou mesmo ao coletivo de trabalhadores. Os resultados propiciarão aos serviços públicos municipais, no Sul do Brasil, subsídios para intervenções (Pieper, Schröer, \& Eilerts, 2019) que favoreçam a segurança e a produção de ambientes saudáveis de trabalho, da mesma forma que a publicação de suas evidências propiciará discussões e contribuições na comunidade científica preocupada com a saúde do trabalhador.

\section{Conclusão}

Os afastamentos laborais por adoecimento foram na maioria de servidores professores, do sexo feminino e na faixa etária de maior produtividade laboral. Constatou-se que a quase totalidade dos casos foram anotados por um diagnóstico codificado segundo a CID-10, não obstante, tenham casos justificados com até seis diagnósticos. Considerando o enquadramento das doenças nos grupos da CID-10, foi predominante o código do grupo (Z00-Z99), referente aos "Fatores que Influenciam o Estado de Saúde e o Contato com os Serviços de Saúde". Neste contexto laborativo, salienta-se que recorrentes afastamentos por adoecimento e agravos podem sinalizar às condições dos ambientes de trabalho e das atividades realizadas por servidores municipais. Espera-se que este estudo venha a contribuir para o desenvolvimento de estratégias de gestão local que visem a segurança e as condições saudáveis dos trabalhadores e dos ambientes laborais.

\section{Agradecimentos}

Agradecemos ao Núcleo de Assistência à Saúde Ocupacional (NASO) e ao Laboratório de Estudos de Processos Socioambientais e Produção Coletiva de Saúde (LAMSA) da Universidade Federal do Rio Grande.

\section{Referências}

Barbosa-Branco, A., Bültmann, U., \& Steenstra, I. (2012). Sickness benefit claims due to mental disorders in Brazil: associations in a population-based study. Cadernos de Saúde Pública, 28(10), 1854-1866. https://doi.org/10.1590/S0102-311X2012001000005

Bryngelson, A., Asberg, M., Nygren, A.., Jensen, I, \& Mittendorfer-Rutz, E. (2013). All-Cause and Cause-Specific Mortality after Long-Term Sickness Absence for Psychiatric Disorders: A Prospective Cohort Study. PloS one, 8(6), e67887. https://doi.org/10.1371/journal.pone.0067887

Brasil (2012). Ministério da Saúde. Conselho Nacional de Saúde. Resolução no 466, de 12 de dezembro de 2012. Aprova as diretrizes e normas regulamentadoras de pesquisa envolvendo seres humanos. http://bvsms.saude.gov.br/bvs/saudelegis/cns/2013/ res0466_12_12_2012.html.

Brasil (2013). Ministério da Saúde. Portaria 1378/2013 que substitui a Portaria 3252/2009. Vigilância em Saúde do Trabalhador. http://www.aids.gov.br/ptbr/legislacao/portaria-no-1378-de-09-de-julho-de-2013.

Brasil (2011). Ministério da Saúde. Secretaria de Vigilância em Saúde. Departamento de Análise de Situação de Saúde. Plano de ações estratégicas para o $\begin{array}{llllll}\text { enfrentamento das doenças crônicas não transmissíveis } & \text { (DCNT) }\end{array}$ https://bvsms.saude.gov.br/bvs/publicacoes/plano_acoes_enfrent_dcnt_2011.pdf

Brasil (2014). Ministério da Saúde. Portaria No 1.271, de 6 de junho de 2014. Define a Lista Nacional de Notificação Compulsória de doenças, agravos e eventos de saúde pública nos serviços de saúde públicos e privados em todo o território nacional, nos termos do anexo, e dá outras providências. Brasília, DF, 2014. http://renastonline.ensp.fiocruz.br/recursos/portaria-1271-6-junho-2014.

Casimirri, E., Vaccari, A., Schito, M., Bonci, M., Stendardo, M., Stefanati, A., Nardini, M., \& Boschetto, P. (2014). Chronic diseases are strongly associated with sickness absences in a sample of Italian public employees. International journal of occupational medicine and environmental health, 27(3), 343-354. https://doi.org/10.2478/s13382-014-0256-x

Cezar-Vaz, M. R. Bonow, C. A., Almeida, M. V.de, Rocha. L. P., \& Borges, A. M. (2015). Mental Health of Elementary Schoolteachers in Southern Brazil: Working Conditions and Health Consequences. The Scientific World Journal, 2015, Article 825925. https://doi.org/10.1155/2015/825925

Costa, R. A. (2017). Absenteísmo por doenças em docentes do ensino fundamental. Dissertação de Mestrado, Faculdade de Medicina de Ribeirão Preto, Universidade de São Paulo, Ribeirão Preto. 10.11606/D.17.2018.tde-25042018-155906. 
Research, Society and Development, v. 10, n. 5, e50810515141, 2021

(CC BY 4.0) | ISSN 2525-3409 | DOI: http://dx.doi.org/10.33448/rsd-v10i5.15141

Costa, G. (2010). Shift work and health: current problems and preventive actions. Safety and health at work, 1(2), 112-123. https://doi.org/10.5491/SHAW.2010.1.2.112

Furlan, A. D., Gnam, W. H., Carnide, N., Irvin, E., Amick, B. C., 3rd, DeRango, K., McMaster, R., Cullen, K., Slack, T., Brouwer, S., \& Bültmann, U. (2012). Systematic review of intervention practices for depression in the workplace. Journal of occupational rehabilitation, 22(3), 312-321. https://doi.org/10.1007/s10926-011-9340-2

IBM Corp (2013). Released. IBM SPSS Statistics for Windows. IBM Corp.

Leão, A. L. M., Barbosa-Branco, A., Turchi, M. D., Steenstra, I. A., \& Cole, D. C. (2017). Sickness absence among municipal workers in a Brazilian municipality: a secondary data analysis. BMC Res Notes, 10, Article 773 https://doi.org/10.1186/s13104-017-3116-5

Leão, A. L. de M., Barbosa-Branco, A., Rassi Neto, E., Ribeiro, C. A. N., \& Turchi, M. D. (2015). Absenteísmo-doença no serviço público municipal de Goiânia. Revista Brasileira de Epidemiologia, 18(1), 262-277. https://dx.doi.org/10.1590/1980-5497201500010020

Muschalla, B., \& Linden, M (2013). Different workplace-related strains and different workplace-related anxieties in different professions. Journal of occupational and environmental medicine, 55(8), 978-982.

https://doi.org/10.1097/JOM.0b013e3182972f04

Pieper, C., Schröer, S., \& Eilerts, A. L. (2019). Evidence of Workplace Interventions-A Systematic Review of Systematic Reviews. International journal of environmental research and public health, 16(19), 3553. https://doi.org/10.3390/ijerph16193553

Organização Mundial da Saúde (OMS) (2006). Universidade de São Paulo - USP. Faculdade de Saúde Pública - FSP. Centro Brasileiro de Classificação de Doenças; Brasil. Ministério da Saúde - MS. Secretaria Executiva. Departamento de Informática do SUS - DATASUS. http://tabnet.datasus.gov.br/cgi/sih/mxcid101m.htm

Reis, R. J., Utzet, M., La Rocca, P. F., Nedel, F. B., Martín, M., \& Navarro, A. (2011). Previous sick leaves as predictor of subsequent ones. International archives of occupational and environmental health, 84(5), 491-499. https://doi.org/10.1007/s00420-011-0620-0

Santos, A. C. de Q., Limongi, J. E., Jorge, M. L. M. P, Jorge, M. T., Pereira, B. B., \& Jorge, P. T. (2015). Aposentadorias por invalidez e Doenças Crônicas entre os servidores da Prefeitura Municipal de Uberlândia, Minas Gerais, 1990-2009. Cadernos Saúde Coletiva, 23(1), 57-62. https://doi.org/10.1590/1414$462 \times 201500010010$

Santos, J. P. dos, \& Mattos, A. P. de. (2010). Absentismo-doença na prefeitura municipal de Porto Alegre, Rio Grande do Sul, Brasil. Revista Brasileira de Saúde Ocupacional, 35(121), 148-156. https://doi.org/10.1590/S0303-76572010000100016

Theorell, T., Hammarström, A., Aronsson, G., Träskman Bendz, L., Grape, T., Hogstedt, C., Marteinsdottir, I., Skoog, I., \& Hall, C. (2015). A systematic review including meta-analysis of work environment and depressive symptoms. BMC public health, 15, 738. https://doi.org/10.1186/s12889-015-1954-4

Wang, M. J., Mykletun, A., Møyner, E. I., Øverland, S., Henderson, M., Stansfeld, S., Hotopf, M., \& Harvey, S. B. (2014). Job strain, health and sickness absence: results from the Hordaland Health Study. PloS one, 9(4), e96025. https://doi.org/10.1371/journal.pone.0096025

Vieira, S (2011). Bioestatística tópicos avançados. Elsevier,

Virtanen, P., Siukola, A., Lipiäinen L., Liukkonen V., Pentti, J., \& Vahtera, J. (2017). Trajectory analyses of sickness absence among industrial and municipal employees, Occupational Medicine, 67(2), 109-113, https://doi.org/10.1093/occmed/kqw104

Vuorio, T., Suominen, S., Kautiainen, H., \& Korhonen, P. (2019). Determinants of sickness absence rate among Finnish municipal employees. Scandinavian journal of primary health care, 37(1), 3-9. https://doi.org/10.1080/02813432.2019.1568710 